\title{
Brillouin Light Scattering Investigations of Magnetic and Elastic Properties in MBE Grown Trilayer Mo/Co/Au Systems
}

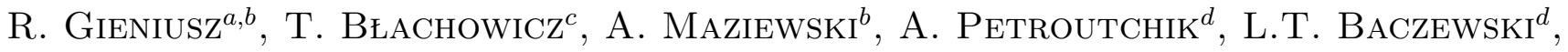

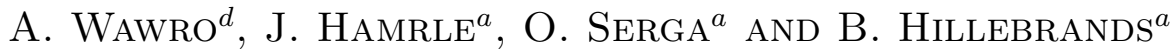 \\ ${ }^{a}$ Fachbereich Physik, Technische Universität Kaiserslautern \\ Erwin-Schrödinger-Str. 56, 67663 Kaiserslautern, Germany \\ ${ }^{b}$ Laboratory of Magnetism, Department of Physics, University of Białystok \\ Lipowa 41, 15-424 Białystok, Poland \\ ${ }^{c}$ Department of Electron Technology, Institute of Physics \\ Silesian University of Technology, Krzywoustego 2, 44-100 Gliwice, Poland \\ ${ }^{d}$ Institute of Physics, Polish Academy of Sciences \\ al. Lotników 32/46, 02-668 Warsaw, Poland
}

\begin{abstract}
The magnetic and elastic properties of epitaxially grown $\mathrm{Mo} / \mathrm{Co} / \mathrm{Au}$ trilayer systems were investigated by the Brillouin light scattering and a ferromagnetic resonance. Using both experimental methods the perpendicular uniaxial anisotropy and the effective in-plane twofold anisotropy contributions were determined as the function of the cobalt thicknesses. Acoustic phonons analysis shows that the elastic constants are decreased with increasing cobalt layer thicknesses.
\end{abstract}

PACS numbers: 76.50.+g, 75.70.Cn, 75.80.+q

\section{Introduction}

Ultrathin layered magnetic systems are important for nowadays applications in the field of magnetoelectronics. They operate as components of spin-valves in magnetic field sensors and magnetic memories [1-3]. Usually, in order to obtain a full picture of the appearing effects, several experimental techniques must be applied simultaneously. Among many possible choices the Brillouin light scattering (BLS) technique from magnetostatic spin-waves [4] and ferromagnetic resonance (FMR) [5] are examples of such combined techniques. Both methods probe the sample at saturation, i.e. without disturbing domain structure. Especially, in BLS experiments, the surface spin-wave, so-called Damon-Eshbach (DE) mode, is usually observed [6,7]. The advantage of BLS spectroscopy is also that it enables observations of acoustic phonons providing information on elastic features. In that sense BLS spectroscopy gives supportive explanations for the magnetic specimen, where the elastic strains take place. The FMR method, on the other hand, provides fast and precise information on magnetic properties of magnetic ultrathin layers.

Co deposited on Mo is an interesting system because of recently reported results of magnetooptical investigations [8]. Influence of buffers and overlayers on magnetic properties of ultrathin cobalt layer has been also studied by FMR [9] in a limited Co thickness range. The aim of this work was to investigate the thickness dependence of the magnetic anisotropy and elastic properties of the $\mathrm{Mo} / \mathrm{Co} / \mathrm{Au}$ trilayer superlattices. The samples were investigated by BLS regarding their magnetic and elastic properties, and, additionally, by FMR in order to confirm effects of anisotropy modifications influenced by the different ferromagnetic layer thicknesses.

\section{Experimental}

The $\mathrm{Al}_{2} \mathrm{O}_{3} / \mathrm{Mo}(20 \mathrm{~nm}) / \mathrm{Co}(d) / \mathrm{Au}(8 \mathrm{~nm}) \quad(d=$ 0.8-31 nm) samples were prepared in a molecular beam epitaxy (MBE) system under ultrahigh vacuum conditions of $10^{-10}$ Torr. The growth process and the chemical composition were monitored in situ by RHEED and Auger spectroscopy, respectively. The sapphire wafers oriented in $(11 \overline{2} 0)$ direction were used as substrates, on which the Mo buffer layer was deposited at $1000^{\circ} \mathrm{C}$ temperature. The cobalt layer was evaporated using an electron gun and the $\mathrm{Au}$ layer from an effusion cell at rates lower than $0.5 \AA / \mathrm{s}$. The BLS measurements of acoustic Rayleigh and Sezawa modes [6] and spin waves (Fig. 1) were performed using a standard Sandercock-type tandem $3+3$-pass spectrometer [10]. The samples were in- 
vestigated in backscattering geometry with the $0.13 \mathrm{~T}$ magnetic field applied in the sample plane. Different angles of incidence were set in order to obtain dispersion relations. The samples were rotated in-plane in steps of $10^{\circ}$ in order to check in-plane asymmetries. FMR experiments were also carried out using the X-band spectrometer.

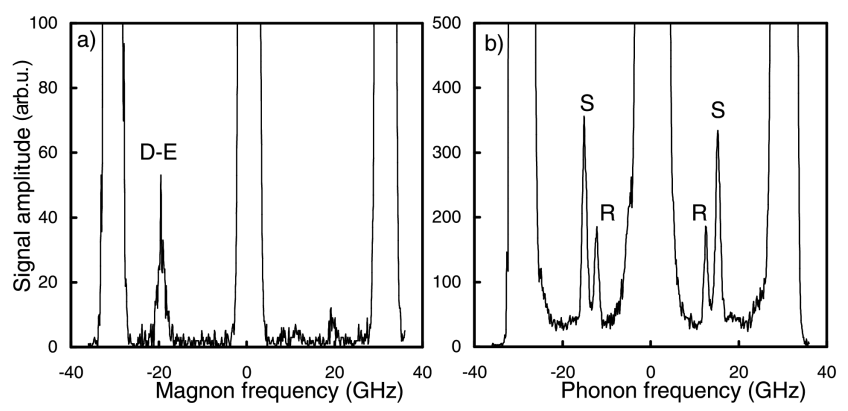

Fig. 1. Typical BLS spectra obtained from experiments with magnons (a) and phonons (b). Descriptions: D-E - the surface Damon-Eshbach mode, R - the surface Rayleigh mode, S - the Sezawa mode. No qualitative differences in spectra, for different samples, were observed.

\subsection{Magnetic properties}

The twofold symmetry for the spin waves were confirmed by fits to BLS data. The BLS fitting was carried out using the well-known formula for the DE mode $[7,11]$. The following phenomenological formula for the free anisotropy energy density was applied [9]:

$$
E_{\text {ani }}=K_{\text {in }} \sin ^{2}\left(\phi-\phi_{\text {ref }}\right) \sin ^{2} \theta+K_{\mathrm{u}} \sin ^{2} \theta,
$$

where $K_{\text {in }}$ is the effective anisotropy constant describing twofold in-plane symmetry, $K_{\mathrm{u}}$ is the perpendicular uniaxial anisotropy constant, $\phi_{\text {ref }}$ is the position of anisotropy easy direction, $\theta$ and $\phi$ are angles defining magnetization polar, and azimuthal positions, respectively. The determined anisotropy constants are given in Table I.

TABLE I

The anisotropy constants obtained from the Brillouin light scattering measurements, for the two samples of different thickness of the Co layer.

\begin{tabular}{c|c|c}
\hline \hline Sample & $K_{\text {in }}\left[\mathrm{J} / \mathrm{m}^{3}\right]$ & $K_{\mathrm{u}}\left[\mathrm{J} / \mathrm{m}^{3}\right]$ \\
\hline $\mathrm{Al}_{2} \mathrm{O}_{3} / \mathrm{Mo}(20 \mathrm{~nm})$ & & \\
$/ \mathrm{Co}(10 \mathrm{~nm}) / \mathrm{Au}(8 \mathrm{~nm})$ & $1.3(1) \times 10^{4}$ & $3.6(1) \times 10^{5}$ \\
$\mathrm{Al}_{2} \mathrm{O}_{3} / \mathrm{Mo}(20 \mathrm{~nm})$ & & \\
$/ \mathrm{Co}(31 \mathrm{~nm}) / \mathrm{Au}(8 \mathrm{~nm})$ & $7.1(6) \times 10^{3}$ & $1.15(1) \times 10^{6}$
\end{tabular}

From the obtained numerical values the changes of the two energy contributions are determined (Eq. (1)). The constant $K_{\mathrm{u}}$ increases by a factor of about 3.2 , while $K_{\text {in }}$ decreases by a factor of about 1.8 , upon a change of the Co layer thickness from $10 \mathrm{~nm}$ to $31 \mathrm{~nm}$.
Additionally, the FMR measurements were also carried out on the set of samples containing the Co layers from $0.8 \mathrm{~nm}$ value up to $31 \mathrm{~nm}$ thickness range. The ultrathin region of thicknesses was very difficult for BLS experiments due to the low scattering cross-section. The resonance field $H_{\mathrm{r}}\left(\phi_{H}\right)$ was measured for different external magnetic field orientations defined by the azimuthal an-

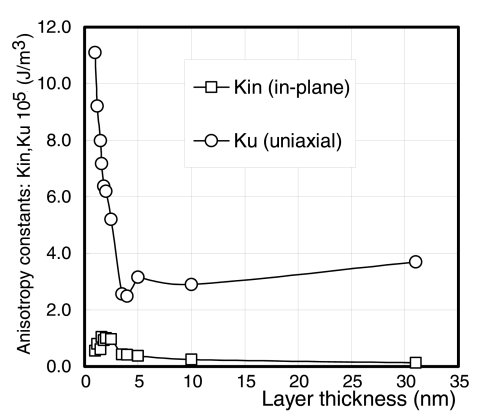

Fig. 2. The in-plane $K_{\text {in }}$ and the uniaxial $K_{\mathrm{u}}$ anisotropy constants as a function of Co layer thickness obtained from the FMR measurements. Solid lines are guides for an eye only.

gle $\phi_{H}$. The twofold symmetry of the resonance field was found for all measured samples when the magnetic field was applied in the sample plane. The values of the $K_{\mathrm{u}}$ and $K_{\text {in }}$ constants were determined from the dependence of the resonance field $H_{\mathrm{r}}\left(\phi_{H}\right)$ depicted in Fig. 2, which shows the anisotropy constants dependence on Co layer thickness for a set of samples - also below the $10 \mathrm{~nm}$ thickness. The FMR results confirmed qualitatively the BLS data pointing on uniaxial anisotropies for ultrathin magnetic layer.

\subsection{Elastic properties}

The BLS experiment revealed that the samples were acoustically isotropic in-plane, however the significant change of the measured acoustic wave velocities was additionally measured for different acoustic wave vectors (Figs. $3 \mathrm{a}-\mathrm{c}$ ) and thicknesses of $1.2 \mathrm{~nm}, 10 \mathrm{~nm}$ and $31 \mathrm{~nm}$. The obtained modifications of the Rayleigh and Sezawa modes, seen in Figs. 3a-c, were confirmed by the estimation of the elastic constants using the so-called fully isotropic approximation (only pure sagittal motions included) [12]. The two independent parameters were then taken as the two elastic constants $c_{11}$ and $c_{12}$. They determine the tensor of the elastic constants by the isotropy conditions: $c_{11}=c_{22}=c_{33}, c_{12}=c_{13}=c_{23}$, $c_{44}=0.5\left(c_{11}-c_{12}\right)=c_{55}=c_{66}$. The reason for the use of this approach is that the crystallographic symmetry of the layers is rather complicated, and in the case of Co layer, this could be a mixture of hcp and fcc phases. 

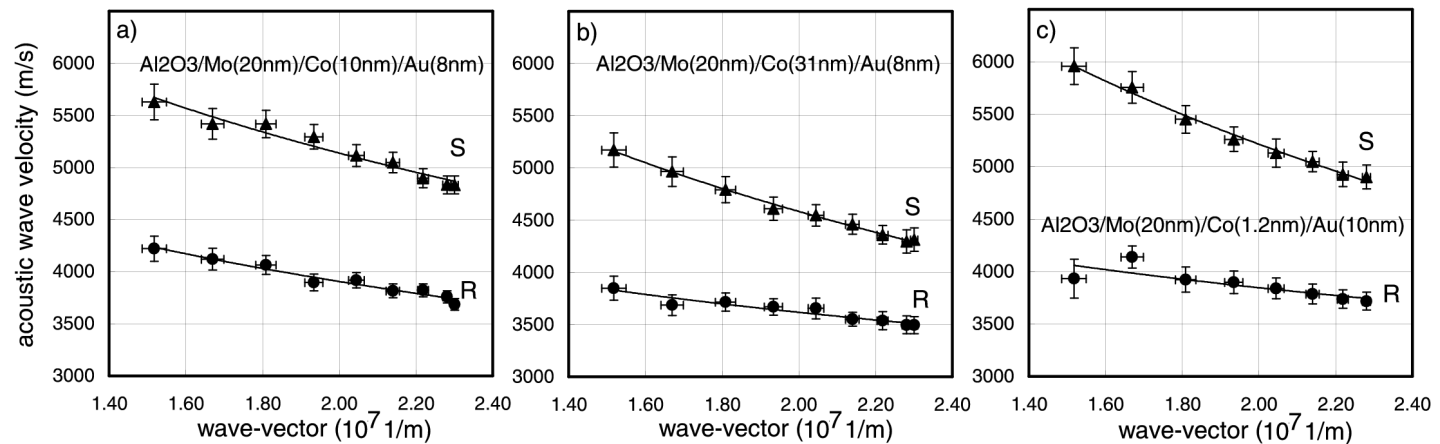

Fig. 3. Experimental results from the Brillouin light scattering on Rayleigh (R) and Sezawa (S) acoustic waves (acoustic phonons): (a) $10 \mathrm{~nm}$ of Co, (b) $31 \mathrm{~nm}$ of Co, (c) $1.2 \mathrm{~nm}$ of Co and $10 \mathrm{~nm}$ of Au overlayer.

Additionally, we assumed that Mo elastic constants were unchangeable for all tested samples, since the Mo layer thickness was kept constant. Similarly, for the same Au layer thicknesses the constants were kept unchangeable. This enabled a multiparameter fitting for BLS experimental data. Thus, the approximate constants can be treated as the effective ones. The values of elastic constants are given in Table II.

TABLE II

Elastic constants values (GPa) obtained from fitting to the Brillouin light scattering measurements. In the brackets Co layer thicknesses are given. For the $d_{\text {Co }}=1.2 \mathrm{~nm}$ case the Au overlayer thickness was equal to $10 \mathrm{~nm}$.

\begin{tabular}{l|c|c|c|c}
\hline \hline $\begin{array}{l}\text { Elastic } \\
\text { constants }\end{array}$ & $\begin{array}{c}\text { Substrate } \\
{[13]}\end{array}$ & Mo & Co & $\mathrm{Au}$ \\
\hline$c_{11}(1.2 \mathrm{~nm})$ & 496 & $440 \pm 15$ & $384 \pm 13$ & $170 \pm 6$ \\
$c_{12}$ & 168 & $221 \pm 7$ & $222 \pm 7$ & $148 \pm 5$ \\
$c_{44}$ & 148 & $110 \pm 8$ & $81 \pm 8$ & $11 \pm 4$ \\
\hline$c_{11}(10 \mathrm{~nm})$ & 496 & $440 \pm 15$ & $326 \pm 11$ & $175 \pm 6$ \\
$c_{12}$ & 168 & $221 \pm 7$ & $209 \pm 7$ & $158 \pm 5$ \\
$c_{44}$ & 148 & $110 \pm 8$ & $65 \pm 7$ & $8.5 \pm 4$ \\
\hline$c_{11}(31 \mathrm{~nm})$ & 496 & $440 \pm 15$ & $254 \pm 8$ & $175 \pm 6$ \\
$c_{12}$ & 168 & $221 \pm 7$ & $121 \pm 4$ & $158 \pm 5$ \\
$c_{44}$ & 148 & $110 \pm 8$ & $66 \pm 4$ & $8.5 \pm 4$
\end{tabular}

\section{Conclusions}

The magnetic anisotropy from BLS and FMR experiments, and elastic properties from BLS measurements were obtained in samples of different Co ferromagnetic layer thicknesses, adjacent to two different nonmagnetic materials.

The perpendicular uniaxial anisotropy was increased while the in-plane twofold anisotropy was decreased for the Co thicknesses larger than $5 \mathrm{~nm}$.
The elastic contribution to the total energy, probed through the acoustic phonon frequencies, seems to be a more complicated issue, since the elastic properties of the system, as a whole, are influenced by all the elastic parameters of all the subsequent layers. The BLS experiment reveals that measured samples were acoustically in-plane isotropic. The estimation of the elastic constants was done using the so-called isotropic approach. The $c_{11}$ and $c_{12}$ elastic constants are reduced with increase in the Co layer from $1.2 \mathrm{~nm}$ to $31 \mathrm{~nm}$. Additionally, the influence of the $\mathrm{Au}$ top-layer onto the acoustic phonons frequencies is observed. These facts can be understood in terms of the $\mathrm{c}_{44}$ elastic constants values, for the layers, which determines the shear wave velocities. The available data for these values [14] clearly show that Co is acoustically faster (about 4 times) than $\mathrm{Au}$, and also the Mo layer is faster than the Co layer, and thus the Co layer operates like an acoustic waveguide for the Rayleigh and Sezawa modes.

\section{Acknowledgments}

We thank O. Gaier, for her assistance in BLS measurements and helpful discussions. This work was supported by the Marie Curie Fellowships "Transfer of Knowledge" (NANOMAG-LAB No. 2004-003177).

\section{References}

[1] T.J. Gafron, S.L. Burkett, S.E. Russek, IEEE Trans. Magn. 36, 2611 (2000).

[2] H. Kanai, K. Noma, J. Hong, J. Fujitsu Sci. Tech. 37, 174 (2001).

[3] S. Kaka, J.P. Nibarger, S.E. Russek, N.A. Stutzke, S.L. Burkett, J. Appl. Phys. 93, 7539 (2003).

[4] B. Hillebrands, J. Hamrle, in: Handbook of Magnetism and Advanced Magnetic Materials, Eds. H. Kronmüller, S.P. Parkin, Vol. 3, Wiley-Interscience, UK 2007, p. 1566.

[5] K. Baberschke, in Ref. [4], p. 1617.

[6] T. Błachowicz, M.H. Grimsditch, in: Encyclopedia of Condensed Matter Physics, Eds. G. Bassani, G. Liedl, P. Wyder, Elsevier Ltd, UK, 2005, p. 199. 
[7] B. Hillebrands, Phys. Rev. B 41, 530 (1990).

[8] A. Stupakiewicz, Z. Kurant, A. Maziewski, L.T. Baczewski, A. Maneikis, A. Wawro, J. Magn. Magn. Mater. 290-291, 242 (2005).

[9] R. Gieniusz, L.T. Baczewski, Z. Kurant, A. Maziewski, A. Wawro, J. Magn. Magn. Mater. 310, 2198 (2007).

[10] R. Mock, B. Hillebrands, J.R. Sandercock, J. Phys. E: Sci. Instrum. 20, 656 (1987).

[11] T. Błachowicz, A. Tillmanns, M. Fraune, R. Ghadimi, B. Beschoten, G. Güntherodt, Phys. Rev. B 75, 054425 (2007).
[12] G.W. Farnell, E.L. Adler, in: Physical Acoustics - Principles and Methods, Eds. W.P. Mason, R.N. Thurston, Vol. 9, Academic Press, New York 1972 , p. 35.

[13] Sapphire elastic constants taken from Crystan Ltd catalog.

[14] M. Grimsditch, R. Bhadra, I.K. Schuller, Phys. Rev. Lett. 58, 1216 (1987). 